\title{
ChemComm
}

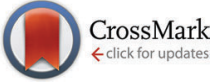

Cite this: Chem. Commun., 2015, 51, 8097

Received 15th January 2015, Accepted 6th April 2015

DOI: $10.1039 / \mathrm{c5cc00401b}$

www.rsc.org/chemcomm

\section{Agglutination of single catalyst particles during fluid catalytic cracking as observed by X-ray nanotomography $\dagger$}

\author{
F. Meirer, ${ }^{a}$ S. Kalirai, ${ }^{a}$ J. Nelson Weker, ${ }^{\text {b }}$ Y. Liu, ${ }^{\mathrm{b}}$ J. C. Andrews ${ }^{\mathrm{b}}$ and \\ B. M. Weckhuysen ${ }^{\star a}$
}

\begin{abstract}
Metal accumulation at the catalyst particle surface plays a role in particle agglutination during fluid catalytic cracking.
\end{abstract}

One of the most important processes in petroleum refining is fluid catalytic cracking (FCC), which is used to convert the heavy hydrocarbon fractions in crude oil into lighter, more valuable products, such as gasoline and propylene. During FCC the heavy, long-chain hydrocarbons are vaporized and 'cracked' into short-chain fractions by a catalyst, or more precisely by billions of tiny, fairly spherical catalyst particles with diameters ranging from 50-150 $\mu \mathrm{m}$. These multi-component catalyst particles consist of a mixture of matrix (i.e., amorphous silica and alumina as well as clay) and zeolite. ${ }^{1}$ To maximize their efficacy a design that considers effects at all length scales is required: from the particle ensemble behaviour in the FCC plant (meters) to the pore network of individual catalyst particles (nanometers). ${ }^{2}$ The latter, for example, is a key factor for the catalytic performance of the FCC catalyst because pore clogging through coke formation or metal deposition can seriously hinder feedstock molecule diffusion into the catalyst, where the heavy hydrocarbon fractions are cracked. Metal intrusion (mainly Fe, $\mathrm{Ni}$, and V) happens through contact with metal-containing feedstock and, in the case of $\mathrm{V}$, can also cause the destruction of the catalytically active phase (zeolite). ${ }^{3}$ While this 'metal poisoning' affects catalyst performance at the single particle level, reduced catalyst performance can also be caused by particle agglutination where, as will be shown in this communication, the deposited metal oxide seems to act as glue.

This conclusion is based on a detailed X-ray nanotomography study of a complete group of agglutinated FCC particles (identified as 'cluster' in the following), consisting of two particles agglutinated

\footnotetext{
${ }^{a}$ Inorganic Chemistry and Catalysis group, Debye Institute for Nanomaterials Science, Utrecht University, Utrecht, The Netherlands.

E-mail: b.m.weckhuysen@uu.nl

${ }^{b}$ Stanford Synchrotron Radiation Lightsource, Stanford University, Menlo Park, CA, USA

$\dagger$ Electronic supplementary information (ESI) available: Supporting information, Movies S1 and S2. See DOI: 10.1039/c5cc00401b
}

to a smaller one, which appears to be a catalyst fragment. The cluster was obtained from a batch of industrial E-cat (equilibrium catalyst) harvested from the regenerator unit of a commercial FCC plant. Analysis of the 3D elemental distributions of $\mathrm{Fe}$ and $\mathrm{Ni}$ indicated that those two metals (and/or their oxides), which are mainly deposited at the outer surface of the particles, seem to act as anchoring points leading to catalyst particle agglutination. The three parts of the cluster showed strong adhesion to each other and were never separated during handling and analysis. This strong adhesion rules out electrostatic forces as reason for agglutination and is in line with the surprising fact that the cluster did not break during the stressful FCC process.

X-ray nanotomography is a powerful tool for the nondestructive 3D analysis of single catalyst particles ${ }^{4,5}$ and Fig. 1(a-e) shows the transmission X-ray microscopy setup at beamline 6-2c at the Stanford Synchrotron Radiation Lightsource. The instrument provides sub-30 $\mathrm{nm} 2 \mathrm{D}$ resolution ${ }^{6}$ and allows mosaic imaging by stitching together multiple fields of view (FOV) of $30 \times 30 \mu^{2}$ to extend the total FOV of the microscope. ${ }^{7}$ This enabled a tomographic scan of the complete cluster, covering a total volume of $54 \times 56 \times 91.5 \mu^{3}$ with a voxel size of $32 \times 32 \times 32 \mathrm{~nm}^{3}$. However, to account for (i) the known loss in $3 \mathrm{D}$ resolution compared to the $2 \mathrm{D}$ resolution of the projection images (an estimated factor of 2-4), ${ }^{8}$ (ii) possible small misalignment effects when stitching the relatively large number of mosaic tiles, and (iii) possible small misalignment between data sets recorded at different energies, the voxel size of the final 3D data was increased to $320 \times 320 \times 320 \mathrm{~nm}^{3}$. Although the real 3D resolution was assumed to be better than $320 \mathrm{~nm}$, this voxel size was found sufficient and allowed a precise and reliable alignment of the data collected below and above the X-ray absorption edges of $\mathrm{Fe}$ and $\mathrm{Ni}$ in order to obtain the 3D relative elemental concentration distributions by differential contrast imaging (see ref. 9 and ESI $\dagger$ for details). Panel (f) in Fig. 1 shows an optical microscopy image of the cluster as mounted in the Kapton ${ }^{\mathrm{TM}}$ capillary $(100 \mu \mathrm{m}$ width and $20 \mu \mathrm{m}$ wall thickness). The two larger particles appeared white in color, while the smaller, connecting particle appeared completely black. 


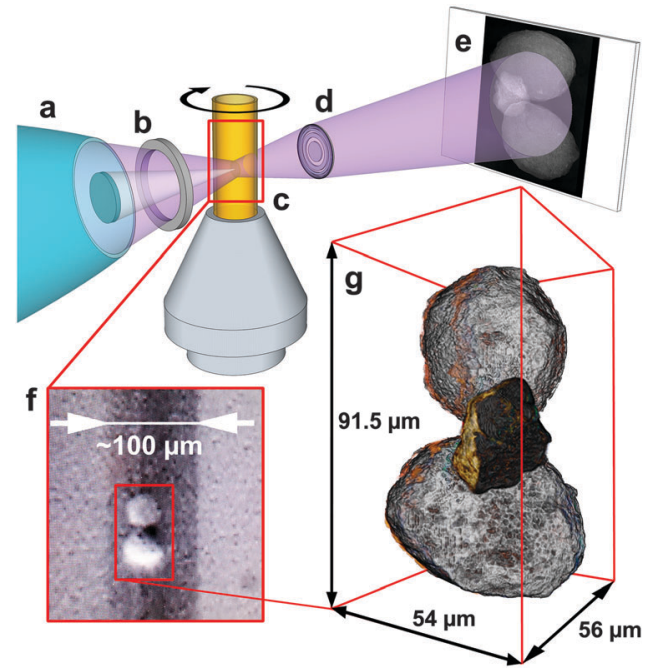

Fig. 1 Top: hard $X$-ray nanotomography setup using the transmission $\mathrm{X}$-ray microscope. Monochromatic $\mathrm{X}$-rays are focused by an elliptical capillary optic (a) through a pinhole (b) illuminating a field of view of $\sim 30 \times 30 \mu \mathrm{m}^{2}$. The $\mathrm{X}$-rays pass through the sample mounted in a Kapton ${ }^{\mathrm{TM}}$ capillary that is fixed on the sample stage (c), which can be moved in the $x, y$, and $z$ direction, and rotated by $360^{\circ}$. A Fresnel zone plate lens (d) projects the sample image onto a scintillator screen (e), where a CCD camera records it. (f) Optical microscopy image of the sample in the Kapton ${ }^{\mathrm{TM}}$ capillary. (g) Rendering of the tomography data. The orange and blue color maps represent relative Fe and $\mathrm{Ni}$ concentrations, respectively (see also Movie S1, ESI†).

Panel (g) of Fig. 1 displays a rendering of the corresponding X-ray nanotomography data, including the relative elemental distributions of Fe (orange color map) and Ni (blue color map).

Fig. 2 shows a single slice through the 3D data, reporting in the top panel the optical density (OD) recorded at $7100 \mathrm{eV}$,
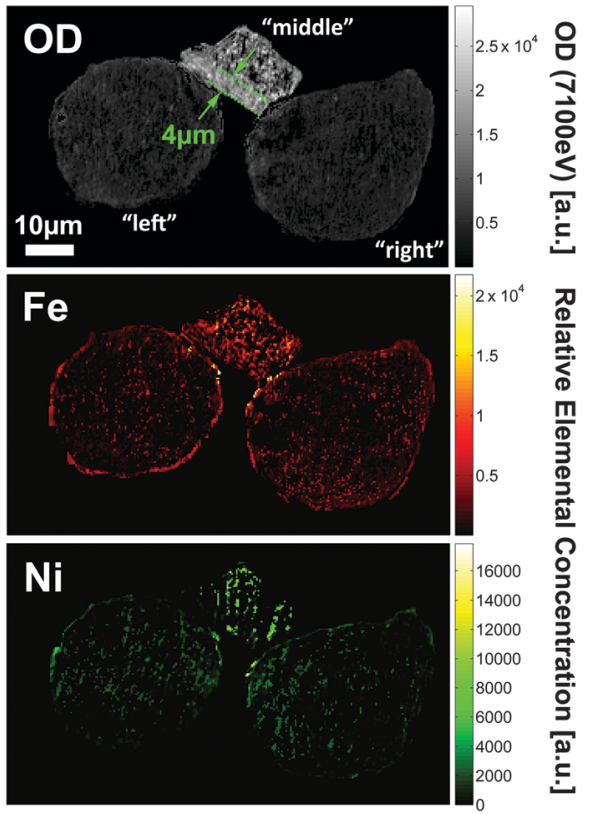

Fig. 2 Slice through the tomography data of the particle conglomerate showing the OD recorded at $7100 \mathrm{eV}$ (top), as well as the relative Fe (center) and $\mathrm{Ni}$ elemental concentrations (bottom). which is used to determine the morphology of the cluster because the absorption at this energy is non-preferential to any of the metals under investigation. The central and bottom panels display the relative Fe and Ni concentrations as obtained by differential absorption contrast imaging (see ref. 9 and ESI $\dagger$ for details). Besides the significantly higher OD of the connecting particle (denoted by 'middle particle' in the following), it shows a clearly different morphology than the other two fairly spherical particles, which resemble typical FCC catalyst particles with porous body and a slightly denser and more compact crust of $\sim 1 \mu \mathrm{m}$ thickness. The middle particle contains an $\sim 4 \mu \mathrm{m}$ thick dense and compact zone in the lower left of the particle (indicated by the green dotted lines in Fig. 2) while the rest of the volume shows a pore structure similar to the body of the two other particles. This suggests that the middle particle is a fragment of another previously intact catalyst particle that broke apart during the FCC process. Usually such debris is removed in the cyclone of the FCC plant, however, it is reasonable to assume that some fragments survive the separation by becoming agglutinated to other intact catalyst particles. The middle particle also shows a very different concentration and $3 \mathrm{D}$ distribution of Fe and Ni than the other two particles (denoted as 'left particle' and 'right particle' in the following; based on the alignment used in Fig. 2 the left particle is the top particle in Fig. 1). While the two large particles contain similar relative $\mathrm{Fe}$ and Ni concentrations when averaged over the entire particle, metal concentrations are significantly higher in the middle particle.

Fig. 3 compares the average OD (at $7100 \mathrm{eV}$ ), and the average relative $\mathrm{Fe}$ and $\mathrm{Ni}$ concentrations of each particle. The average OD and Fe concentration in the middle particle is a factor of $\sim 3$ larger than in both other particles, while the relative Ni concentration was found to be 4.5 and 5.5-fold higher than in the right and left particle, respectively. While Fig. 2 suggests a homogenous distribution of both metals in the middle particle, there is a clear accumulation of $\mathrm{Fe}$ and (less pronounced) $\mathrm{Ni}$ at the surface of the other particles. This is expected for E-cat particles, based on earlier $2 \mathrm{D}^{10,11}$ and $3 \mathrm{D}^{4}$ studies. In the latter work the authors performed an analysis of the radial dependence of $\mathrm{Fe}$ and $\mathrm{Ni}$ for the whole E-cat particle, showing that the metal accumulation is highly localized and mainly restricted to the first 1-2 $\mu \mathrm{m}$ of the particle surface.

Here we performed a radial evaluation for each of the three particles, assessing possible differences in the way each particle

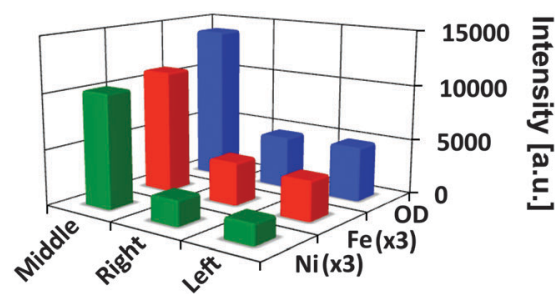

Fig. 3 Average optical density (OD) and average relative elemental concentrations of $\mathrm{Fe}$ and $\mathrm{Ni}$ for each part of the particle conglomerate; indicated by 'left', 'right', and 'middle', corresponding to the alignment used in Fig. 2. Fe and Ni relative concentrations have been scaled by a factor of 3 for clarity. 

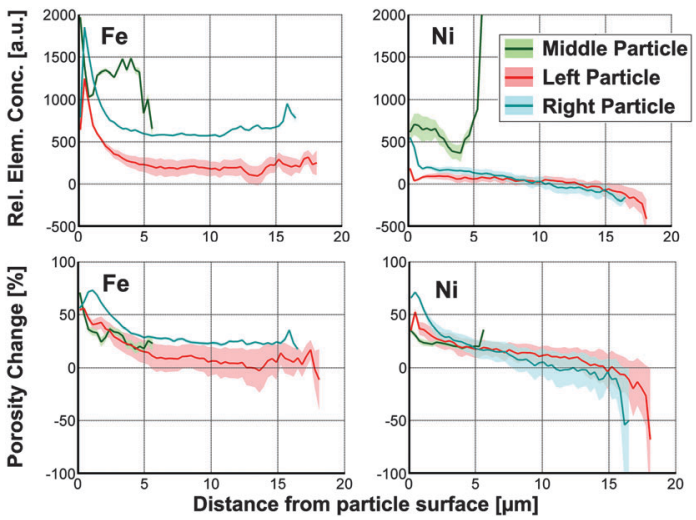

Fig. 4 Radial Fe and Ni relative concentrations of each part of the particle conglomerate. The parts are indicated 'left' (red), 'right', (cyan) and 'middle' (green) corresponding to the alignment used in Fig. 2.

accumulated Fe and Ni. For this evaluation voxels with identical distances to the particle surface were pooled, forming concentric 'shells' of single voxel thickness $(320 \mathrm{~nm})$. Thus, this method accounts for any irregular shape of the particle and allows plotting the average relative $\mathrm{Fe}$ and $\mathrm{Ni}$ concentrations of each shell as a function of distance from the particle surface. ${ }^{4,12}$ Furthermore, the porosity of each shell can be determined as the ratio of empty space in the shell to the total volume of the shell. This enables a correlation of porosity changes with the presence of the analysed metal, quantifying how the metals are clogging the macro-pore space. ${ }^{4}$

The resulting radial distributions (Fig. 4) show that the larger particles show a typical radial dependence of relative Fe and Ni concentrations, decreasing abruptly within the first $\sim 2 \mu \mathrm{m}$ from the particle surface. The right particle shows larger relative concentrations and pore blocking effects, suggesting that it accumulated more $\mathrm{Fe}$ and $\mathrm{Ni}$ during its lifetime; i.e. it is 'catalytically older', than the left particle. The middle particle shows no clear radial dependence, but rather a similar metal distribution for each radial shell. The extreme relative $\mathrm{Ni}$ concentration in the most central shells of the middle particle indicates heterogeneity of the metal distribution. Because most of the Ni in the middle particle is present as 'hot-spots' (highly localized large elemental concentrations) that are averaged over the small central shells, which probe a relatively small volume of the particle, this can cause significantly larger average $\mathrm{Ni}$ concentrations. Fig. 4 confirms that the relative $\mathrm{Ni}$ and $\mathrm{Fe}$ concentrations are very similar in the middle particle (see Fig. 3), while Fe has a clearly larger relative concentration than $\mathrm{Ni}$ in the two other particles. The high Ni concentration of the middle particle further suggests that it is the 'catalytically oldest' part, because $\mathrm{Ni}$ is not present in the original catalyst particle matrix and can only be accumulated during FCC. This supports the hypothesis that this part is a fragment of a (catalytically) older, collapsed and then shattered E-cat particle.

The strong difference in OD between the middle and the two other particles allows a clear identification of each part (Fig. 2) and suggests that just a thin surface layer acts as 'glue', joining the three parts of the cluster. Such a 'sticky' surface layer could be related to the enhanced Fe and Ni loading at the surface. This hypothesis is supported by the observation that the interfaces of the particles contain many mutual and localized areas of highest relative $\mathrm{Fe}$ and $\mathrm{Ni}$ concentrations (Fig. 2). This in turn agrees with a note made in an earlier 2D study, suggesting that the interface region between two joined E-cat particles was enriched in Fe when compared to other surface regions. ${ }^{11}$ In order to quantify our observation we analysed the average relative $\mathrm{Fe}$ and $\mathrm{Ni}$ concentrations in each interface region and compared them to the average surface concentrations (Fig. 5). The interface regions were defined as particle surface layers of $640 \mathrm{~nm}$ (2-voxel thickness), limited to the contact area(s) of each particle. The corresponding volumes are displayed in magenta, cyan, and yellow in Fig. 5, indicating the evaluated particle sub-volumes of the left (or top), middle, and right particle, respectively. The average particle surface concentrations were calculated by averaging the relative concentrations of the first two surface shells from the radial evaluation displayed in Fig. 4, i.e. averaging the relative concentrations of $\mathrm{Fe}$ and $\mathrm{Ni}$ in a $640 \mathrm{~nm}$ thick surface layer.

The results show clearly that the average concentrations at the interfaces of the two larger particles are higher than the average concentrations over the whole surface. While relative Fe interface concentrations increased by a factor of 2 and 1.6 for the left (top) and right particle, respectively, corresponding relative Ni concentrations were enhanced by a factor of 4.5 and 2 . For both full particles the relative Fe concentrations at the interface were even larger than that in the middle particle, while relative Ni concentrations at the interface were found to be similar in all particles. The middle particle did not show significant differences between relative concentrations in the contact areas and the overall particle surface. Because Fe is part of the particle matrix we cannot exclude higher Fe concentrations at possibly pre-existing interfaces of pristine FCC particles, however, the fact that both larger particles show high Ni loading

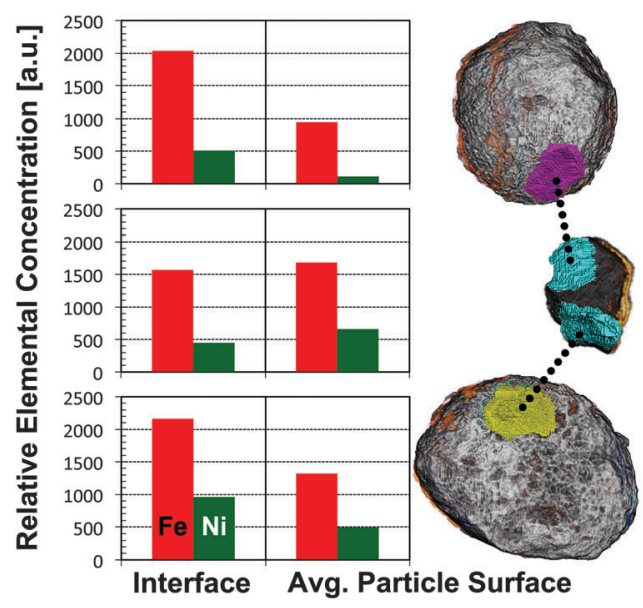

Fig. 5 Evaluated interface regions of the agglutinated particles and corresponding averaged relative $\mathrm{Fe}$ and $\mathrm{Ni}$ concentrations of each subvolume (in magenta, cyan, yellow for the left, middle and right particles, respectively), compared with the average metal concentrations in the respective particle surface. 
at the interface implies that agglutination happened after both particles spent some time in the FCC cycle. The metal loading at the interface is even larger than elsewhere, suggesting a higher surface concentration where the particles joined and that the middle particle later protected the surface from abrasion, preserving the state at the time of conglutination.

Besides particle size distribution, density, and attrition resistance, particle clustering is an important parameter that affects catalyst fluidity in the FCC unit. Studies of the fluidization behaviour of particulates including FCC catalysts have concluded that not only physical properties such as particle density, size, shape, and roughness, but also interparticle forces influence their fluidization, ${ }^{13,14}$ especially in the case of E-cat particles. ${ }^{15}$ The nature and interplay of these cohesive interparticle forces is not yet completely understood, and appears to be different for different environments (e.g. bubbling fluidized beds, risers, or liquid jets). ${ }^{14}$ McMillan et al. ${ }^{14}$ suggested that particle clustering during FCC might be due to coulombic forces, van der Waals forces, cohesive bridging, and drag-induced hydrodynamic forces. However, these forces cannot explain the strong chemical interaction observed here, joining the particles even after removal from the FCC unit. Such permanent binding could be explained by the fact that $\mathrm{Fe}$, especially together with $\mathrm{Na}$, can lower the melting point of silica leading to vitrification of the particle surface. ${ }^{16}$ If a particle with high metal loading (like the middle particle) has a molten surface it can cluster with other particles forming a strong chemical bond at the interface. If many particles in the unit accumulate such large metal concentrations, particle clustering could be enhanced through cohesive bridging, as a result of the molten or near molten surface on the particles. ${ }^{14}$

With this first high-resolution 3D study of the concentration distribution of $\mathrm{Fe}$ and $\mathrm{Ni}$ in a system of agglutinated catalyst particles we were able to confirm not only enhanced $\mathrm{Fe}$ and $\mathrm{Ni}$ concentrations on the surface of E-cat particles ${ }^{4}$ but also that interfaces between agglutinated E-cat particles contain Fe and $\mathrm{Ni}$ concentrations above the average surface concentrations. ${ }^{11}$ These observations suggest that the surface accumulation of metals could in fact be responsible for the enhanced interparticle forces observed for E-cat particles that lead to increased particle clustering, and hence decreased activity. ${ }^{15}$

This work was supported by the Netherlands Research School Combination-Catalysis (NRSC-C) and a European Research Council (ERC) Advanced Grant (no. 321140). The Stanford Synchrotron Radiation Lightsource is a directorate of SLAC National
Accelerator Laboratory and an Office of Science User Facility operated for the U.S. Department of Energy Office of Science by Stanford University.

\section{Notes and references}

1 (a) J. Scherzer, Appl. Catal., 1991, 75, 1-32; (b) Y. Chen, in Handbook of Fluidization and Fluid-Particle Systems, ed. W.-C. Yang, Marcel Dekker, New York, 2003, ch. 14; (c) R. Sadeghbeigi, Fluid Catalytic Cracking Handbook, Elsevier Science, Oxford, 2012, ch. 4.

2 (a) I. L. C. Buurmans and B. M. Weckhuysen, Nat. Chem., 2012, 4, 873-886; (b) J. D. Grunwaldt and C. G. Schroer, Chem. Soc. Rev., 2010, 39, 4741-4753; (c) J. D. Grunwaldt, J. B. Wagner and R. E. Dunin-Borkowski, ChemCatChem, 2013, 5, 62-80.

3 (a) R. Pompe, S. Järås and N. Vannerberg, Appl. Catal., 1984, 13, 171-179; (b) K.-J. Chao, L.-H. Lin, Y.-C. Ling, J.-F. Hwang and L.-Y. Hou, Appl. Catal., 1995, 121, 217-229; (c) E. Kugler and D. Leta, J. Catal., 1988, 395, 387-395.

4 (a) F. Meirer, D. T. Morris, S. Kalirai, Y. Liu, J. C. Andrews and B. M. Weckhuysen, J. Am. Chem. Soc., 2015, 137, 102-105; (b) F. Meirer, S. Kalirai, D. Morris, S. Soparawalla, Y. Liu, G. Mesu, J. C. Andrews and B. M. Weckhuysen, Sci. Adv., 2015, 1, e1400199, DOI: 10.1126/ sciadv.1400199.

5 (a) J. C. da Silva, K. Mader, M. Holler, D. Haberthür, A. Diaz, M. Guizar-Sicairos, W.-C. Cheng, Y. Shu, J. Raabe, A. Menzel and J. A. van Bokhoven, ChemCatChem, 2015, 7, 413-416; (b) S. R. Bare, M. E. Charochak, S. D. Kelly, B. Lai, J. Wang and Y. K. Chen-Wiegart, ChemCatChem, 2014, 6, 1427-1437; (c) A. M. Beale, S. D. M. Jacques and B. M. Weckhuysen, Chem. Soc. Rev., 2010, 39, 4656-4672.

6 (a) Y. Liu, J. C. Andrews, J. Wang, F. Meirer, P. Zhu, Z. Wu and P. Pianetta, Opt. Express, 2011, 19, 540-545; (b) J. C. Andrews and B. M. Weckhuysen, ChemPhysChem, 2013, 14, 3655-3666.

7 Y. Liu, F. Meirer, P. A. Williams, J. Wang, J. C. Andrews and P. Pianetta, J. Synchrotron Radiat., 2012, 19, 281-287.

8 (a) M. Holler, A. Diaz, M. Guizar-Sicairos, P. Karvinen, E. Färm, E. Härkönen, M. Ritala, A. Menzel, J. Raabe and O. Bunk, Sci. Rep., 2014, 4, 3857; (b) N. Banterle, K. H. Bui, E. A. Lemke and M. J. Beck, J. Struct. Biol., 2014, 183, 363-367.

9 (a) Y. Liu, F. Meirer, J. Wang, G. Requena, P. Williams, J. Nelson, A. Mehta, J. C. Andrews and P. Pianetta, Anal. Bioanal. Chem., 2012, 404, 1297-1301; (b) T. L. Kao, C. Y. Shi, J. Wang, W. L. Mao, Y. Liu and W. Yang, Microsc. Res. Tech., 2013, 76, 1112-1117.

10 A. C. Psarras, E. F. Iliopoulou, L. Nalbandian, A. A. Lappas and C. Pouwels, Catal. Today, 2007, 127, 44-53.

11 O. Bayraktar and E. L. Kugler, Catal. Lett., 2003, 90, 155-160.

12 F. Yang, Y. Liu, S. K. Martha, Z. Wu, J. C. Andrews, G. E. Ice, P. Pianetta and J. Nanda, Nano Lett., 2014, 14, 4334-4341.

13 (a) J. Shabanian and J. Chaouki, Chem. Eng. J., 2015, 259, 135-152; (b) J. R. Royer, D. J. Evans, L. Oyarte, Q. Guo, E. Kapit, M. E. Möbius, S. R. Waitukaitis and H. M. Jaeger, Nature, 2009, 459, 1110-1113.

14 J. McMillan, F. Shaffer, B. Gopalan, J. W. Chew, C. Hrenya, R. Hays, S. B. R. Karri and R. Cocco, Chem. Eng. Sci., 2013, 100, 39-51.

15 P. Lettieri, J. G. Yates and D. Newton, Powder Technol., 2000, 110, 117-127.

16 G. Yaluris, W.-C. Cheng, M. Peters, L. T. McDowell and L. Hunt, in Studies in Surface Science and Catalysis, ed. M. Occelli, Elsevier B.V., New York, 2004, vol. 149, pp. 139-163. 